\title{
P04-3-21 Poster session
}

\section{Prorrenin/renin receptor expression in immune cells in an ovariectomized rats model}

\section{Maria E Hernandez-Campos, Nestor A Torres-Frayre, Octavio Rodriguez-Cortes, Pedro Lopez- Sanchez}

Seccion de Estudios de Posgrado e Investigacion, Escuela Superior de Medicina del Instituto Politecnico Nacional, Mexico

Estrogen is a sex steroid hormone important in women, predominantly synthesized in the ovaries and released into the bloodstream. Estrogen is a regulator of inflammation; exerting a number of known anti-inflammatory effects through a variety of different mechanisms, both genomic and non-genomic. On the other hand, the renin-angiotensin system (RAS) has vasoconstrictor and proinflammatory functions that regulate cardiovascular homeostasis. Most of the proinflammatory effects are mediated by AT1 receptor, which activates proinflammatory signaling pathways including the transcription factor NFkappaB activation. The prorenin and renin receptor (PRR) is the newest component of RAS and one of its signaling pathways include NFkappaB activation. Estrogen has an inhibitory effect on RAS. To study the effect of estrogen absence upon PRR expression in immune cells from spleen, we used an ovariectomized rats (OVX) model. We validated the model using vaginal histology and morphometric measurements. We used flow cytometry to analyze splenic immune cells, PRR expression and pro- and antiinflamatory citokines. To evaluate inflammatory response in these conditions, we stimulated a group with LPS injection to OVX and control rats. We found an increase in PRR expression and function, measured as PLZF expression, in whole spleen cells from OVX rats, Differential expression, however, was found in macrophages (M), T lymphocytes (TL), and NK cells in these rats: A clear decrease in CD3+LT and M, whereas NK cells showed a slight increase. After $24 \mathrm{~h}$ of LPS injection, the PRR expression pattern increased uniformly in all studied cells, with an increase in PLZF expression. Our data indicate that PRR in immune cells is estrogen-controlled and this expression modifies activity of this receptor, suggesting a role in inflammatory response. 